\title{
Correction to "A Study on Pharmacokinetics of Bosentan with Systems Modeling, Part 1: Translating Systemic Plasma Concentration to Liver Exposure in Healthy Subjects"
}

\begin{abstract}
In the above article [Li R, Niosi M, Johnson N, Tess DA, Kimoto E, Lin J, Yang X, Riccardi KA, Ryu S, El-Kattan AF, Maurer TS, Tremaine LM, and Di L (2018) Drug Metab and Dispos 46 (4) 346-356; DOI: https://doi.org/10.1124/dmd.117.078790], there is an error in Figure 5 caption with the second word 'total' should have been 'unbound'. A correction published in April 2018 noted a compositor error correctly changed to $1 / \mathrm{nM} / \mathrm{h}$. The HTML and PDF versions of the article now have been updated with the corrected Figure 5 caption as provided below.
\end{abstract}

Fig. 5. (A-I) Simulated unbound systemic and liver tissue concentrations (A, D, and G), ratios between unbound liver tissue and unbound systemic plasma concentrations (B, E, and $\mathrm{H}$ ), and induction effects of bosentan (C, F, and I). Red and blue curves in subplots (A), (D), and (G) represent systemic and liver concentrations, respectively. Red and blue curves in subplots (C), F), and (I) represent induction effects in enterocytes and the liver, respectively. The solid lines and shaded areas represent the median and 95\% CI of the simulations generate with parameter values identified in Markov chain Monte Carlo. BID, twice daily; QD, once daily.

The authors regret this error and apologize for any inconvenience this may have caused. 